\title{
Mixing-demixing in a trapped degenerate fermion-fermion mixture
}

\author{
Sadhan K. Adhikari* \\ Instituto de Física Teórica, UNESP—São Paulo State University, 01.405-900 São Paulo, São Paulo, Brazil
}

(Received 1 February 2006; published 27 April 2006)

\begin{abstract}
We use a time-dependent dynamical mean-field-hydrodynamic model to study mixing-demixing in a degenerate fermion-fermion mixture (DFFM). It is demonstrated that with the increase of interspecies repulsion and/or trapping frequencies, a mixed state of a DFFM could turn into a fully demixed state in both threedimensional spherically symmetric as well as quasi-one-dimensional configurations. Such a demixed state of a DFFM could be experimentally realized by varying an external magnetic field near a fermion-fermion Feshbach resonance, which will result in an increase of interspecies fermion-fermion repulsion, and/or by increasing the external trap frequencies.
\end{abstract}

DOI: $10.1103 /$ PhysRevA.73.043619

PACS number(s): $03.75 . S s$

\section{INTRODUCTION}

Recent successful observation of a degenerate bosonfermion mixture (DBFM) of trapped alkali-metal atoms by different experimental groups [1-4] initiated intensive experimental studies of different novel phenomena [5-7]. Among these experiments, apart from the study of the DBFMs ${ }^{6,7} \mathrm{Li}[3],{ }^{23} \mathrm{Na}-{ }^{6} \mathrm{Li}[4]$, and ${ }^{87} \mathrm{Rb}-{ }^{40} \mathrm{~K}[5,6,8]$, there have been studies of degenerate fermion-fermion mixtures (DFFMs) of two components of ${ }^{40} \mathrm{~K}[1]$ and ${ }^{6} \mathrm{Li}[2,7]$. The collapse in a DBFM of ${ }^{87} \mathrm{Rb}^{40} \mathrm{~K}$ atoms has been observed [5] and studied [9] by Modugno et al. and by the present author [10] and more recently by Ospelkaus et al. [8]. There have also been investigations on the formation of bright [11] and dark [12] solitons in a DBFM.

Several theoretical investigations [13-15] of a trapped DBFM studied the phenomenon of mixing-demixing when the boson-fermion repulsion is increased. For a weak bosonfermion repulsion at $0 \mathrm{~K}$ both the Bose-Einstein condensate (BEC) and the degenerate Fermi gas (DFG) have maxima of probability density at the center of the harmonic trap. However, with the increase of boson-fermion repulsion, the maximum of the probability density of the DFG could be slowly expelled from the central region. With further increase of boson-fermion repulsion, the DFG could be completely expelled from the central region which will house only the BEC. This phenomenon has been termed mixing-demixing in a DBFM. The phenomenon of demixing has drawn some attention lately as in a demixed state an exotic configuration of the mixture is formed, where there is practically no overlap between the two components and one can be observed and studied independently of the other. It has been argued [15] that such a demixed state in a DBFM should be possible experimentally by increasing the interspecies scattering length near a Feshbach resonance. In view of this it is of interest to see if, in addition to a DBFM, such a demixed state appears in a DFFM.

The purpose of this paper is to study and illustrate this mixing-demixing phenomenon in a trapped DFFM using a

\footnotetext{
*Electronic address: adhikari@ift.unesp.br; URL: http:// www.ift.unesp.br/users/adhikari/index.html
}

coupled time-dependent mean-field-hydrodynamic model inspired by the success of a similar model in the investigation of fermionic collapse [10] and bright [11] and dark [12] solitons in a DBFM. The conclusions of the study on bright solitons [11] are in agreement with a microscopic study [16], and those on collapse [10] are in agreement with experiments $[5,8]$. There have been prior suggestions of mixing-demixing in a trapped DFFM upon an increase of interspecies repulsion. Roth and Feldmeier [17] discuss the possibility of demixing mathematically using an energy density functional approach. Amoruso et al. [18] demonstrate demixing numerically using model hydrodynamic flow equations. In contrast to these previous time-independent studies for stationary states, the present study relies on a time-dependent formulation and demonstrates demixing also for an increase of the trapping frequencies and should be considered complementary to these previous investigations. The present suggestion of demixing with an increase of trapping frequency is easier to implement experimentally than increasing the interspecies scattering length using a Feshbach resonance.

We look for mixing-demixing in a trapped DFFM in the spherically symmetric as well as quasi-one-dimensional configurations. For a weak fermion-fermion repulsion, we find the formation of a fully mixed state with both the fermion clouds occupying the central region of the trap. If the fermion numbers of the two types of fermions are greatly different, a fully demixed state of the DFFM appears with the increase of fermion-fermion repulsion, when the component with smaller number of fermions is completely expelled from the central region of the trap, which is occupied only by the component with the larger number of fermions. This demixing also appears for increasing trap frequency, which increases the fermion density and hence fermion-fermion repulsion, thus resulting in a demixing. To the best of our knowledge this is the first study of mixing-demixing in a DFFM using a time-dependent formulation capable of investigating nonequilibrium states. Previous studies of demixing $[17,18]$ employed time-independent formulations appropriate for stationary states only.

In Sec. II we present the mean-field-hydrodynamic model we use in our investigation in the spherically symmetric as well as quasi-one-dimensional configurations. The numerical results are presented in Sec. III where we illustrate demixing 
with the increase of interspecies repulsion and of the harmonic trapping potential. Finally, in Sec. III a brief summary of our findings are given.

\section{MEAN-FIELD-HYDRODYNAMIC MODEL}

A proper description of a DFFM should employ $[15,19]$ a fully antisymmetrized microscopic Slater determinant wave function for each component. However, a simplified meanfield-hydrodynamic Lagrangian for a DFG has been used successfully to study a DBFM $[11,12]$, which we shall use in the present investigation. The virtue of the mean-field model over a microscopic description is its simplicity and good predictive power. To develop a set of practical timedependent mean-field-hydrodynamic equations for a DFFM, we consider the following Lagrangian density $[11,12]$ :

$$
\begin{aligned}
\mathcal{L}= & \frac{i}{2} \hbar \sum_{j=1,2}\left(\Psi_{j} \frac{\partial \Psi_{j}^{*}}{\partial t}-\Psi_{j}^{*} \frac{\partial \Psi_{j}}{\partial t}\right)+g_{12} n_{1} n_{2} \\
& +\sum_{j=1}^{2}\left(\frac{\hbar^{2}\left|\nabla \Psi_{j}\right|^{2}}{6 m_{j}}+V_{j} n_{j}+\frac{3}{5} A_{j} n_{j}^{5 / 3}\right),
\end{aligned}
$$

where $m_{j}$ is the mass of component $j(=1,2), \Psi_{j}$ a complex probability amplitude, $n_{j}=\left|\Psi_{j}\right|^{2}$ the real probability density, $N_{j} \equiv \int d \mathbf{r} n_{j}(\mathbf{r})$ the number, $A_{j}=\hbar^{2}\left(6 \pi^{2}\right)^{2 / 3} /\left(2 m_{j}\right)$, and $V_{j}$ the confining trap. Here the interspecies coupling is $g_{12}$ $=2 \pi \hbar^{2} a_{12} / m_{R}$ with the reduced mass $m_{R}=m_{1} m_{2} /\left(m_{1}+m_{2}\right)$, and $a_{12}$ the interspecies scattering length. The interaction between intraspecies fermions in spin-polarized state is highly suppressed due to the Pauli blocking term $3 A_{j} n_{j}^{5 / 3} / 5$ and has been neglected in Eq. (1) and will be neglected throughout. The kinetic energy terms in this equation $\hbar^{2}\left|\nabla \Psi_{j}\right|^{2} /\left(6 m_{j}\right)$ are derived from a hydrodynamic equation for the fermions [20] and contribute little to this problem compared to the dominating Pauli blocking term in Eq. (1). However, the inclusion of the kinetic energy terms in Eq. (1) leads to a smooth solution for the probability density everywhere [11]. To keep the algebra simple and without losing generality in our calculation we shall take equal fermion masses: $m_{1}=m_{2} \equiv m / 3$. This simulates well the fermion mixtures of ground and excited states of ${ }^{6} \mathrm{Li}[2]$ and ${ }^{40} \mathrm{~K}[1]$ atoms observed experimentally. The Lagrangian density of each fermion component in Eq. (1) is identical to that used in Refs. [11,12].

The mean-field dynamical equations for the system are just the usual Euler-Lagrange (EL) equations

$$
\frac{d}{d t} \frac{\partial \mathcal{L}}{\partial\left(\partial \Psi_{j}^{*} / \partial t\right)}+\sum_{k=1}^{3} \frac{d}{d x_{k}} \frac{\partial \mathcal{L}}{\partial\left(\partial \Psi_{j}^{*} / \partial x_{k}\right)}=\frac{\partial \mathcal{L}}{\partial \Psi_{j}^{*}},
$$

where $x_{k}, k=1,2,3$, are the three space components, and $j$ $=1,2$ refer to the fermion components.

With Lagrangian density (1) the following EL equations of motion are derived in a straightforward fashion [10,11]:

$$
\left(-i \hbar \frac{\partial}{\partial t}-\frac{\hbar^{2} \nabla_{\mathbf{r}}^{2}}{6 m_{j}}+V_{j}(\mathbf{r})+A_{j} n_{j}^{2 / 3}+g_{12} n_{k}\right) \Psi_{j}=0,
$$

$$
j \neq k=1,2 .
$$

This is essentially a time-dependent version of a similar time-independent model used recently for fermions [14]. For a stationary state Eqs. (3) yield the same result as the formulation of Ref. [14]. For a system with a large number of fermions both reduce to $[10,14]$ the well-known ThomasFermi approximation $[21,22]: n_{j}=\left[\left(\mu_{j}-V_{j}\right) / A_{j}\right]^{3 / 2}$, with $\mu_{j}$ the chemical potential.

In the spherically symmetric case we take $V_{j}(\mathbf{r})$ $=\frac{1}{2}\left(3 m_{j}\right) \omega^{2} r^{2}$ and $\Psi_{j}(\mathbf{r} ; t)=\psi_{j}(r ; t)$. Now transforming to dimensionless variables defined by $x=\sqrt{2} r / l, \quad \tau=t \omega, l$ $\equiv \sqrt{\hbar /(m \omega)}$ and

$$
\frac{\phi_{j}(x ; \tau)}{x}=\sqrt{\frac{4 \pi l^{3}}{N_{j} \sqrt{8}}} \psi_{j}(r ; t),
$$

we get $[10]$

$$
\left(-i \frac{\partial}{\partial \tau}-\frac{\partial^{2}}{\partial x^{2}}+\frac{x^{2}}{4}+\mathcal{N}_{j j}\left|\frac{\phi_{j}}{x}\right|^{4 / 3}+\mathcal{N}_{j k}\left|\frac{\phi_{k}}{x}\right|^{2}\right) \phi_{j}(x ; \tau)=0,
$$

where $j \neq k=1,2$ and $\mathcal{N}_{j j}=3\left(3 \pi N_{j} / 2\right)^{2 / 3}, \mathcal{N}_{j k}=6 \sqrt{2} N_{k} a_{12} / l$. The normalization of the wave-function components is given by $\int_{0}^{\infty} d x\left|\phi_{j}(x ; \tau)\right|^{2}=1, j=1,2$.

We next reduce Eq. (3) to a minimal quasi-onedimensional form in a cigar-shaped geometry where the confining trap of anisotropy $\nu$ has the form $V_{j}(\mathbf{r})=\frac{1}{2} 3 m_{j} \omega^{2}\left(\rho^{2}\right.$ $+\nu^{2} z^{2}$ ), with $\rho$ the radial vector and $z$ the axial vector. For a cigar-shaped geometry $\nu \ll 1$, we consider solutions of Eq. (3) of the type $\Psi_{j}(\mathbf{r}, t)=\sqrt{N_{j}} \varphi_{j}(z, t) \psi_{j}^{(0)}(\rho)$, where

$$
\left|\psi_{j}^{(0)}(\rho)\right|^{2} \equiv \frac{3 m_{j} \omega}{\pi \hbar} \exp \left(-\frac{3 m_{j} \omega \rho^{2}}{\hbar}\right)
$$

corresponds to the respective circularly symmetric groundstate wave function in the absence of nonlinear interactions and satisfies

$$
-\frac{\hbar^{2}}{6 m_{j}} \nabla_{\rho}^{2} \psi_{j}^{(0)}+\frac{3}{2} m_{j} \omega^{2} \rho^{2} \psi_{j}^{(0)}=\hbar \omega \psi_{j}^{(0)}
$$

with normalization $2 \pi \int_{0}^{\infty}\left|\psi_{j}^{(0)}(\rho)\right|^{2} \rho d \rho=1$. Now the dynamics is carried by $\varphi_{j}(z, t)$ and the radial dependence is frozen in the ground state $\psi_{j}^{(0)}(\rho)$. The separation of the variables is suggested by the structure of Eq. (3).

Averaging over the radial mode $\psi_{j}^{(0)}$, i.e., multiplying Eq. (3) by $\psi_{j}^{(0) *}(\rho)$ and integrating over $\rho$, we obtain the following one-dimensional dynamical equations $[11,12]$ :

$$
\begin{gathered}
\left(-i \hbar \frac{\partial}{\partial t}-\frac{\hbar^{2}}{6 m_{j}} \frac{\partial^{2}}{\partial z^{2}}+\frac{3}{2} m_{j} \nu^{2} \omega^{2} z^{2}+F_{j j} N_{j}^{2 / 3}\left|\varphi_{j}\right|^{4 / 3}\right. \\
\left.+F_{j k} N_{k}\left|\varphi_{k}\right|^{2}\right) \varphi_{j}(z, t)=0,
\end{gathered}
$$

where $j \neq k=1,2$ and 


$$
\begin{gathered}
F_{j k}=g_{12} \frac{\int_{0}^{\infty}\left|\psi_{k}^{(0)}\right|^{2}\left|\psi_{j}^{(0)}\right|^{2} \rho d \rho}{\int_{0}^{\infty}\left|\psi_{j}^{(0)}\right|^{2} \rho d \rho}=g_{12} \frac{M_{12} \omega}{\pi \hbar}, \\
F_{j j}=A_{j} \frac{\int_{0}^{\infty}\left|\psi_{j}^{(0)}\right|^{2+4 / 3} \rho d \rho}{\int_{0}^{\infty}\left|\psi_{j}^{(0)}\right|^{2} \rho d \rho}=\frac{3 A_{j}}{5}\left(\frac{3 m_{j} \omega}{\pi \hbar}\right)^{2 / 3},
\end{gathered}
$$

where $M_{12}=3 m_{1} m_{2} /\left(m_{1}+m_{2}\right)$. In Eq. (8) the normalization is $\int_{-\infty}^{\infty}\left|\varphi_{j}(z, t)\right|^{2} d z=1$.

In Eq. (8), we introduce the dimensionless variables $\tau$ $=t \nu \omega / 2, x=z / l_{z}, \phi_{j}=\sqrt{l_{z}} \varphi_{j}$, with $l_{z}=\sqrt{\hbar /(\nu \omega m)}$, so that

$$
\begin{aligned}
& \left(-i \frac{\partial}{\partial \tau}-\frac{\partial^{2}}{\partial x^{2}}+c^{2} x^{2}+\mathcal{N}_{j k}\left|\phi_{k}\right|^{2}+\mathcal{N}_{j j}\left|\phi_{j}\right|^{4 / 3}\right) \phi_{j}(x, \tau)=0, \\
& j \neq k=1,2,
\end{aligned}
$$

where $c=1, \mathcal{N}_{j k}=12 a_{12}\left(N_{k} / \nu\right) / l_{z}$, and $\mathcal{N}_{j j}=9\left(6 \pi N_{j} / \nu\right)^{2 / 3} / 5$. However, by taking $c \neq 1$ we can modify the harmonic trap. In Eq. (11), the normalization condition is given by $\int_{-\infty}^{\infty}\left|\phi_{j}(x, \tau)\right|^{2} d x=1$.

\section{NUMERICAL RESULTS}

We solve the coupled mean-field-hydrodynamic equations (5) and (11) numerically using a time-iteration method based on the Crank-Nicholson discretization scheme elaborated in [23]. We discretize the mean-field equations using time step 0.001 and space step 0.05 . In the quasi-one-dimensional case we span $x$ from -60 to 60 , and in the three-dimensional case we span $x$ from 0 to 30 . In both cases we start with the Gaussian ground-state solution of the linear harmonic oscillator problem as input at $\tau=0$ while we set the nonlinearities $\mathcal{N}_{j k}=\mathcal{N}_{j j}=0$. In the three-dimensional case governed by Eq. (5) we take $\phi_{j}(x, \tau=0)=(\pi / 2)^{-1 / 4} \exp \left(-x^{2} / 4\right)$ and in the onedimensional case governed by Eq. (11) we take $\phi_{j}(x, \tau=0)$ $=\pi^{-1 / 4} \exp \left(-x^{2} / 2\right)$. With these initial states we perform time evaluation of Eqs. (5) and (11). During the course of time evolution the nonlinear terms $\mathcal{N}_{j k}$ and $\mathcal{N}_{j j}$ are slowly introduced at the rate of 0.0001 at each time step of 0.001 . The solution evolves slowly with time and attains the final stationary configuration after the full desired nonlinearity is introduced slowly. It is important to introduce the nonlinearity slowly to achieve a final stationary configuration.

In our numerical investigation we take $l=1 \mu \mathrm{m}$ and consider the fermions to have the mass of ${ }^{40} \mathrm{~K}$ atoms and to occupy two levels of this fermionic atom. In the spherically symmetric case this corresponds to a radial frequency $\omega$ $\approx 2 \pi \times 83 \mathrm{~Hz}$. Consequently, the unit of time is $1 / \omega$ $\approx 2 \mathrm{~ms}$, and the unit of length $l / \sqrt{2} \approx 0.7 \mu \mathrm{m}$. In the quasione-dimensional case we take $\nu=0.1$ and $l_{z}=1 \mu \mathrm{m}$ corresponding to an axial frequency $\omega \nu \approx 2 \pi \times 83 \mathrm{~Hz}$. Consequently, the unit of time is $2 /(\nu \omega) \approx 4 \mathrm{~ms}$, and the unit of length $l_{z}=1 \mu \mathrm{m}$.
After a small experimentation it is realized that in both Eqs. (5) and (11), demixing is most favored when $N_{1}$ and $N_{2}$ are widely different from each other. Under this condition one of the components is much larger in size than the other and easily expels the smaller component from the center of the trap upon an increase in interspecies repulsion or trap frequency, and we shall consider only this asymmetric configuration here. In previous investigations [18] demixing in the symmetric configuration $\left(N_{1}=N_{2}\right)$ was considered. The interspecies repulsion can be manipulated through the scattering length $a_{12}$ by varying a background magnetic field near a fermion-fermion Feshbach resonance [24].

First, we present results for the quasi-one-dimensional case from a solution of Eq. (11). After some experimentation we take in the initial DFFM $N_{1}=1000, N_{2}=10$, and $a_{12}$ $=50 \mathrm{~nm}, \nu=0.1$. This corresponds to nonlinearities $\mathcal{N}_{11}$ $\approx 5917, \mathcal{N}_{22} \approx 274, \mathcal{N}_{12}=60$, and $\mathcal{N}_{21}=6000$. The two overlapping Gaussian-type fermionic profiles in this case are shown in Fig. 1(a). Next we increase the interspecies repulsion by increasing the interspecies scattering length $a_{12}$. With the increase of repulsion the component with smaller number of fermions is slowly deiven out of the central region which continues to be solely occupied by the component with larger number of fermions. In Figs. 1(b) $-1(\mathrm{~d})$, we plot the results for $a_{12}=100,150$, and $400 \mathrm{~nm}$, from where we find that a complete demixing has occured for $a_{12}=400 \mathrm{~nm}$. In the last case two symmetrical peaks of the component with the smaller number of fermions appear where the density of the component with the larger number of fermions is practially zero. The interesting thing to note is that in Figs. 1(a)-1(d), the profile of the degenerate gas with the larger number of fermions remains practially unchanged, while the weaker component with a much smaller number of fermions is expelled from the central region with the increase of repulsion. The mutual repulsion has practically no effect on the "heavier" component when it expels the "lighter" component from the central region. Had we considered the symmetric case with $N_{1}=N_{2}=1000$ a much larger $a_{12}$ would have been necessary for a complete demixing.

Next we consider demixing in the three-dimensional spherically symmetric case from the solutions of Eq. (5). In this case we take in the initial DFFM $N_{1}=10000, N_{2}=100$, and $a_{12}=50 \mathrm{~nm}$. This corresponds to nonlinearities $\mathcal{N}_{11}$ $\approx 3914, \mathcal{N}_{12} \approx 42, \mathcal{N}_{21} \approx 4243$, and $\mathcal{N}_{22} \approx 181$. The overlapping fermionic quasi-Gaussian profiles of the DFGs in this case are shown in Fig. 2(a). Now we increase the interspecies repulsion by increasing the scattering length $a_{12}$. With the increase of repulsion among the two species, smooth Gaussian profiles of the DFFM are destroyed and the component with smaller number of fermions is slowly driven out of the central region which continues to be occupied by the component with larger number of fermions. In Figs. 2(b)-2(d) we plot the results for $a_{12}=300,400$, and $800 \mathrm{~nm}$, respectively. From Figs. 2 we see that demixing has increased with the increase of repulsion among the two components. In Fig. 2(d) the component with the smaller number of fermions is practically located outside the region occupied by the component with the larger number of fermions.

The dimensionless parameter controlling the validity of the present dilute (weakly interacting) mean-field- 

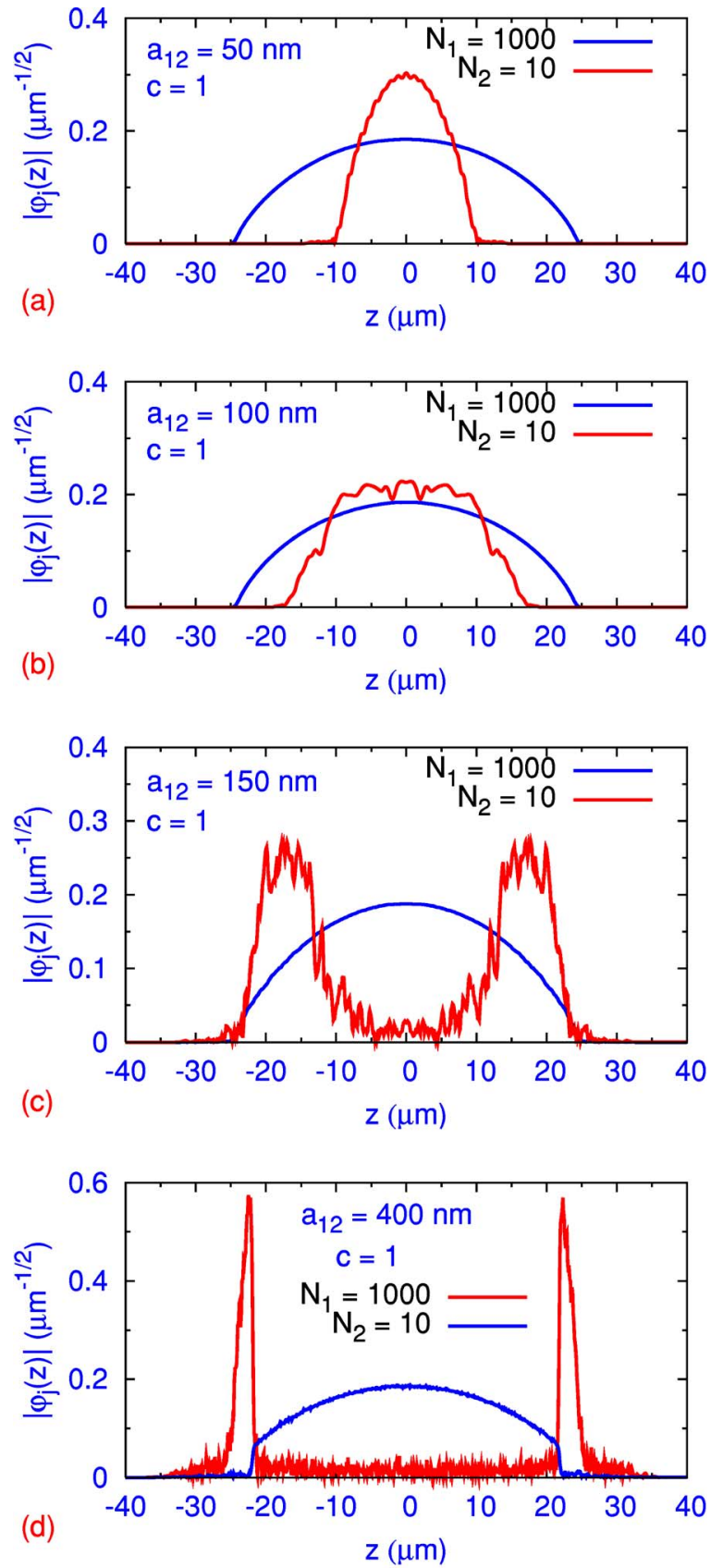

FIG. 1. (Color online) The fermionic profiles $\varphi_{j}(z)$ of Eq. (8) vs $z$ for $N_{1}=1000$ and $N_{2}=10$ in the quasi-one-dimensional case $(\nu$ $=0.1$ ) for $a_{12}=$ (a) 50, (b) 100, (c) 150 , and (d) $400 \mathrm{~nm}$.

hydrodynamic model is the number of particles in a scattering volume $|a|^{3}$, where $a$ is the scattering length. This can be written as $\bar{n}|a|^{3}$, where $\bar{n}$ is the average density of the gas [21]. When $\bar{n}|a|^{3} \ll 1$ the system is said to be dilute or weakly interacting. The opposite limit $\bar{n}|a|^{3} \gg 1$ is known as the unitarity limit. It is interesting to find out if the demixing in the three-dimensional case shown in Fig. 2 occurred in the dilute limit. In Fig. 2(c) demixing is visible for $|a|=400 \mathrm{~nm}$ where there are 10000 atoms in a sphere of radius $R \approx 18 \mu \mathrm{m}$. Consequently, $\quad \bar{n}=10000 /\left(4 \pi R^{3} / 3\right) \approx 0.4 \mu \mathrm{m}^{-3}$ and $|a|^{3}$ $\approx 0.064 \mu \mathrm{m}^{3}$, so that $\bar{n}|a|^{3} \approx 0.025$. So demixing starts in the
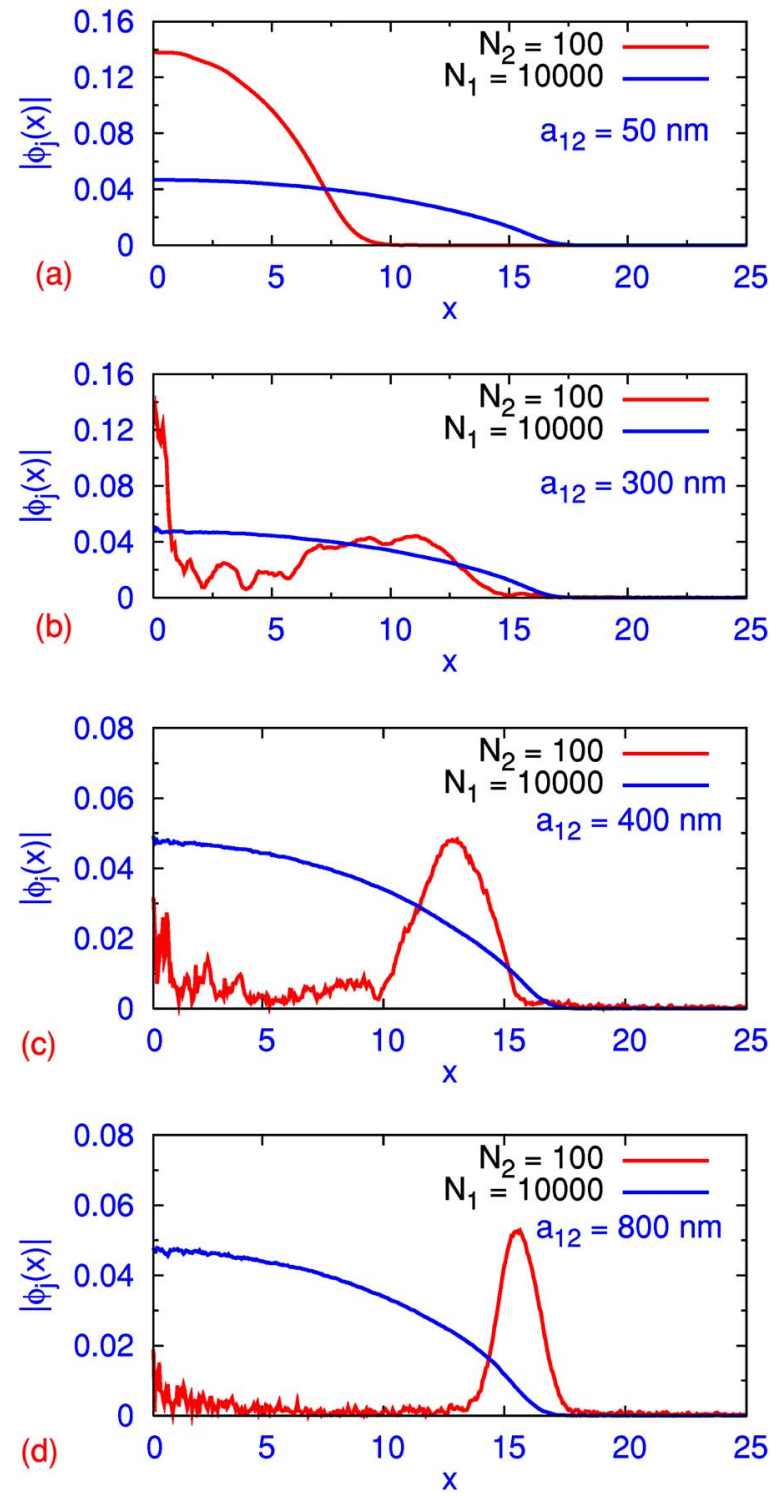

FIG. 2. (Color online) The fermionic profiles $\phi_{j}(x)$ of Eq. (5) vs $x$ in dimensionless units for $N_{1}=10000$ and $N_{2}=100$ in the spherically symmetric case for $a_{12}=$ (a) 50, (b) 300, (c) 400, and (d) $800 \mathrm{~nm}$

dilute weakly interacting limit and continues in the unitarity limit.

The present condition of demixing is consistent with that of Ref. [18], where the authors reported demixing as a function of the parameter [22]

$$
\gamma \equiv \frac{E_{\mathrm{int}}}{E_{\mathrm{pot}}}=\alpha N^{1 / 6} \frac{a}{l},
$$

with $\alpha=2^{1 / 3} 3^{1 / 6}\left(8192 / 2835 \pi^{2}\right) \approx 0.44$ and $N$ the number of atoms, $E_{\text {int }}$ is the interaction energy, and $E_{\text {pot }}$ is the potential energy of the harmonic oscillator trap. The parameter $\gamma$ compares the interaction energy with the potential energy in the harmonic trap and provides a more reliable account of the words "weakly interacting." A small value of the parameter $\gamma$ together with the condition $\bar{n}|a|^{3} \ll 1$ is necessary for the 

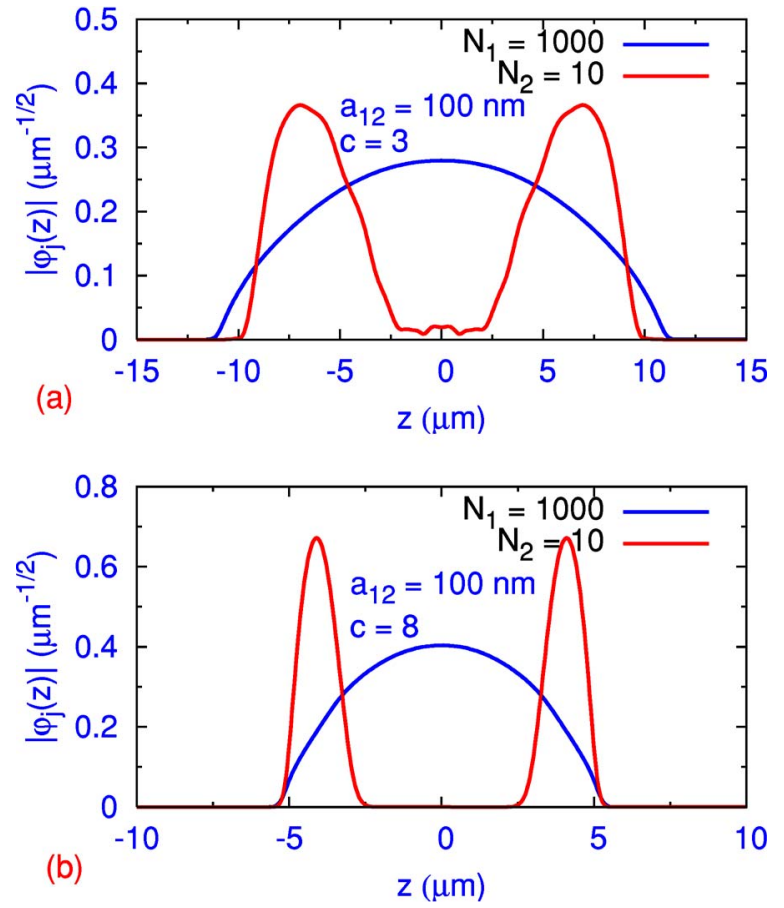

FIG. 3. (Color online) The fermionic profiles $\varphi_{j}(z)$ of Eq. (8) vs $z$ for $N_{1}=1000, N_{2}=10$, and $a_{12}=100 \mathrm{~nm}$ in the quasi-onedimensional case $(\nu=0.1)$ for $c=$ (a) 3 and (b) 8 .

DFFM to be weakly interacting. In the situation of Fig. 2(c) $(a=400 \mathrm{~nm})$ demixing has started for $\gamma \approx 0.44 \times 10000^{1 / 6}$ $\times 0.4 \approx 0.8$ and is absent in the situation of Fig. 2(b) ( $a$ $=300 \mathrm{~nm}$ ) where $\gamma \approx 0.6$. In the study of Ref. [18] demixing started at $\gamma \approx 0.7$, and was absent for $\gamma \approx 0.54$ quite consistent with present finding. The small value of $\gamma(<1)$ obtained in the present study as well as in Ref. [18] confirms the dilute or weakly interacting nature of the DFFM in the demixing limit. However, according to the condition obtained in Ref. [17], demixing should occur only in the unitarity limit. We do not know the reason for this discrepancy. Further investigations are needed for clarification.

Now we investigate the possibility of demixing in a DFFM by increasing the strength of the harmonic trap. We illustrate the results only in the quasi-one-dimensional case described by Eq. (11). From Fig. 1(b) we find that there is no real demixing in the solution of Eq. (11) for $N_{1}=1000, N_{2}$ $=10, a_{12}=100 \mathrm{~nm}$, and $c=1$. In this case we find that demixing appears as we increase $c$ keeping other parameters fixed. The results are illustrated in Figs. 3(a) and 3(b) for $c=3$ and
8. The increase of parameter $c$ increases the trap frequency in the same proportion. From Fig. 3(a) we find that demixing already appears for $c=3$ and increases in Fig. 3(b) for $c=8$. For a large $c$, the appearance of demixing for a smaller $a_{12}$ can be understood from the structure of Eq. (11). An increase of the trapping frequency corresponds to a decrease in the harmonic oscillator length $l_{z}$. Then a smaller value of $a_{12}$ can lead to a larger nonlinearity $\mathcal{N}_{j k}$ as in the definition of $\mathcal{N}_{j k}$, $a_{12}$ is always scaled with respect to $l_{z}$. However, with the increase of the trapping frequency the scaling length $l_{z}$ is reduced and the degenerate gas is confined to a smaller region in space as can be seen from Figs. 3(a) and 3(b).

\section{SUMMARY}

Using a coupled set of time-dependent mean-fieldhydrodynamic equations for a trapped DFFM derived using the Lagrangian density for a DFG suggested recently [11,12], we study the phenomenon of demixing in a trapped DFFM. We show that, for a repulsive interspecies fermion-fermion interaction, demixing is favored in a DFFM with different numbers of fermions. Demixing appears for a large value of interspecies scattering length $a_{12}$ denoting a large repulsion between the components. As the interspecies repulsion increases, the fermionic component with smaller number of fermions is expelled from the central region which is solely occupied by the component with the larger number of fermions. Demixing in a trapped DFFM can be realized experimentally by changing the interspecies scattering length $a_{12}$ to a large positive value by exploiting a fermion-fermion Feshbach resonance [24]. Demixing also appears with the increase of trapping frequency for a smaller $a_{12}$. This latter possibility to achieve demixing is more attractive experimentally as it is easier to increase the trapping frequency than to increase $a_{12}$ near a Feshbach resonance by manipulating a background magnetic field [24]. A proper treatment of DFFM should be performed using a fully antisymmetrized many-body Slater determinant wave function [15] as in the case of atomic and molecular scattering involving many electrons [25]. However, in view of the success of the hydrodynamic model in other contexts [10-12] we do not believe that the present study on mixing-demixing in a DFFM is so peculiar as to have no general validity.

\section{ACKNOWLEDGMENTS}

The work is supported in part by the CNPq and FAPESP of Brazil.
[1] B. DeMarco and D. S. Jin, Science 285, 1703 (1999).

[2] K. M. O’Hara, S. L. Hemmer, M. E. Gehm, S. R. Granade, and J. E. Thomas, Science 298, 2179 (2002).

[3] F. Schreck, L. Khaykovich, K. L. Corwin, G. Ferrari, T. Bourdel, J. Cubizolles, and C. Salomon, Phys. Rev. Lett. 87, 080403 (2001); A. G. Truscott, K. E. Strecker, W. I. McAlexander, G. B. Partridge, and R. G. Hulet, Science 291, 2570
(2001).

[4] Z. Hadzibabic, C. A. Stan, K. Dieckmann, S. Gupta, M. W. Zwierlein, A. Gorlitz, and W. Ketterle, Phys. Rev. Lett. 88, 160401 (2002); Z. Hadzibabic, S. Gupta, C. A. Stan, C. H. Schunck, M. W. Zwierlein, K. Dieckmann, and W. Ketterle, ibid. 91, 160401 (2003).

[5] G. Modugno, G. Roati, F. Riboli, F. Ferlaino, R. J. Brecha, and 
M. Inguscio, Science 297, 2240 (2002).

[6] G. Roati, F. Riboli, G. Modugno, and M. Inguscio, Phys. Rev. Lett. 89, 150403 (2002).

[7] K. E. Strecker, G. B. Partridge, and R. G. Hulet, Phys. Rev. Lett. 91, 080406 (2003).

[8] C. Ospelkaus, S. Ospelkaus, K. Sengstock, and K. Bongs, Phys. Rev. Lett. 96, 020401 (2006).

[9] M. Modugno, F. Ferlaino, F. Riboli, G. Roati, G. Modugno, and M. Inguscio, Phys. Rev. A 68, 043626 (2003).

[10] S. K. Adhikari, Phys. Rev. A 70, 043617 (2004).

[11] S. K. Adhikari, Phys. Rev. A 72, 053608 (2005).

[12] S. K. Adhikari, J. Phys. B 38, 3607 (2005).

[13] Y. Takeuchi and H. Mori, Phys. Rev. A 72, 063617 (2005).

[14] Z. Akdeniz, A. Minguzzi, P. Vignolo, and M. P. Tosi, Phys. Lett. A 331, 258 (2004); P. Capuzzi, A. Minguzzi, and M. P. Tosi, Phys. Rev. A 68, 033605 (2003).

[15] K. Molmer, Phys. Rev. Lett. 80, 1804 (1998).

[16] T. Karpiuk, M. Brewczyk, S. Ospelkaus-Schwarzer, K. Bongs, M. Gajda, and K. Rzażewski, Phys. Rev. Lett. 93, 100401 (2004).

[17] R. Roth and H. Feldmeier, J. Phys. B 34, 4629 (2001).

[18] A. Amoruso, I. Meccoli, A. Minguzzi, and M. P. Tosi, Eur.
Phys. J. D 8, 361 (2000).

[19] R. Roth, Phys. Rev. A 66, 013614 (2002); R. Roth and H. Feldmeier, ibid. 65, 021603(R) (2002); T. Miyakawa, T. Suzuki, and H. Yabu, ibid. 64, 033611 (2001); X.-J. Liu, M. Modugno, and H. Hu, ibid. 68, 053605 (2003).

[20] P. Capuzzi, A. Minguzzi, and M. P. Tosi, Phys. Rev. A 67, 053605 (2003).

[21] F. Dalfovo, S. Giorgini, L. P. Pitaevskii, and S. Stringari, Rev. Mod. Phys. 71, 463 (1999).

[22] L. Vichi and S. Stringari, Phys. Rev. A 60, 4734 (1999).

[23] S. K. Adhikari and P. Muruganandam, J. Phys. B 35, 2831 (2002); P. Muruganandam and S. K. Adhikari, ibid. 36, 2501 (2003).

[24] K. M. O’Hara, S. L. Hemmer, S. R. Granade, M. E. Gehm, J. E. Thomas, V. Venturi, E. Tiesinga, and C. J. Williams, Phys. Rev. A 66, 041401(R) (2002); K. Dieckmann, C. A. Stan, S. Gupta, Z. Hadzibabic, C. H. Schunck, and W. Ketterle, Phys. Rev. Lett. 89, 203201 (2002); T. Loftus, C. A. Regal, C. Ticknor, J. L. Bohn, and D. S. Jin, ibid. 88, 173201 (2002); C. A. Regal, M. Greiner, and D. S. Jin, ibid. 92, 083201 (2004).

[25] P. K. Biswas and S. K. Adhikari, J. Phys. B 33, 1575 (2000); 31, L737 (1998); 31, 3147 (1998); 31, L315 (1998). 